\title{
Recurrent and Troublesome Variceal Bleeding from Parastomal Caput Medusae
}

\author{
Claire Strauss, Malathi Sivakkolunthu and Abraham A. Ayantunde \\ Department of Surgery, Southend University Hospital, Westcliff-on-Sea, UK
}

\begin{abstract}
Variceal bleeding is common in chronic liver disease and is a frequent cause of acute upper gastrointestinal bleeding. The most common site of varices is the lower oesophagus but they may occur at any location where there are portosystemic anastomoses and collateral vascular formation. Location of ectopic varices at the site of enterocutaneous stomas is rare. We report on three cases of recurrent and severe bleeding from parastomal varices, requiring hospital admission. The patients had chronic liver disease but of different aetiological factors. Variceal formation results from portal hypertension due to chronic liver disease. There are various treatment options for parastomal variceal bleeding, including local, medical, and surgical interventions. Management of parastomal variceal bleeding presents a recurring and difficult problem. Bleeding may be considerable and sometimes life threatening. This diagnosis must be considered in patients with chronic liver disease presenting with stomal bleeding, even where the variceal formation may not be readily visible. (Korean J Gastroenterol 2014;64:290-293)
\end{abstract}

Key Words: Stoma; Variceal bleeding; Liver cirrhosis; Propranolol; Portal hypertension

\section{INTRODUCTION}

Variceal bleeding is a common problem in patients with chronic liver disease of any aetiology. The most common site of varices is the lower end of the oesophagus but they may form in any location where there is portosystemic collateral vessel formation. ${ }^{1,2}$ Non gastro-oesophageal (ectopic) varices occur at sites including the rectum, peritoneum, umbilicus (caput medusae), right diaphragm, and falciform and splenic ligaments. ${ }^{1-5}$ Varices at these unusual sites account for $1-5 \%$ of episodes of variceal bleeding., ${ }^{2,4}$ Occurrence of acute bleeding, which may be severe and life threatening is the main reason for the discovery of such ectopic varices. Diagnosis and management of bleeding from these unusual sites is challenging. Therefore, it is important to consider a systematic approach to identification and treatment of patients presenting with this condition.

We report on three patients with chronic liver disease of different aetiological factors with recurrent and troublesome parastomal variceal bleeding and discuss the investigations and the challenging management options. Written informed consent was obtained from the patients for publication of these cases and anonymised accompanying images.

\section{CASE REPORTS}

\section{Case 1}

A 52-year-old woman was admitted to hospital after reporting a significant volume of fresh blood loss from her ileostomy. This was her sixth admission for the same problem. This patient had undergone a Hartmann's procedure for sigmoid diverticular perforation nine years earlier. She subsequently underwent a reversal of Hartmann, which was complicated by severe anastomotic stricture. This anastomotic structuring necessitated the formation of an ileostomy.

She had a history of excess alcohol consumption which had caused compensating alcoholic induced chronic liver disease (liver cirrhosis with Child-Pugh score A) with known

Received February 17, 2014. Revised May 7, 2014. Accepted May 8, 2014

(C) This is an open access article distributed under the terms of the Creative Commons Attribution Non-Commercial License (http://creativecommons.org/licenses/ by-nc/3.0) which permits unrestricted non-commercial use, distribution, and reproduction in any medium, provided the original work is properly cited.

Correspondence to: Abraham A. Ayantunde, Department of Surgery, Southend University Hospital, Prittlewell Chase, Westcliff-on-Sea, SSO ORY, UK.

Tel: +44-1702435555, Fax: +44-1702385856, E-mail: biodunayantunde@yahoo.co.uk

Financial support: None. Conflict of interest: None. 
portal hypertension, oesophago-gastric varices, and hepato-splenomegaly. She had previously been diagnosed with rectal varices which were initially mistaken for haemorrhoids. On clinical examination, a parastomal hernia with abnormal radial vascular formation was detected at the mucocutaneous junction and the surrounding skin showed extensive circumferential purplish discolouration (raspberry appearance) with bruising around the ileostomy (Fig. 1).

In the course of her several hospital admission episodes her haemoglobin levels dropped significantly, requiring blood transfusion. She underwent oesophagogastroduodenoscopy showing non-bleeding oesophageal varices. CT and MRI scans showed features in keeping with chronic liver disease and parastomal hernia. Flexible sigmoidoscopy only showed non-bleeding rectal varices and anastomotic stricture. Enteroscopy via the ileostomy showed no intraluminal cause of the bleeding.

Treatment options used included suture haemostasis and cauterisation of the bleeding vessels at the mucocutaneous junction of the ileostomy with short-lived success. She had a trial with propranolol to the maximum tolerated dose as higher doses were restricted by postural hypotension. She continues to present with self-limiting but less frequent and minor episodes of para-stomal variceal bleeding.

\section{Case 2}

This 48-year-old man was diagnosed with ulcerative colitis at the age of 15 and underwent a panproctocolectomy and an end ileostomy formation at the age of 39. Four years later,

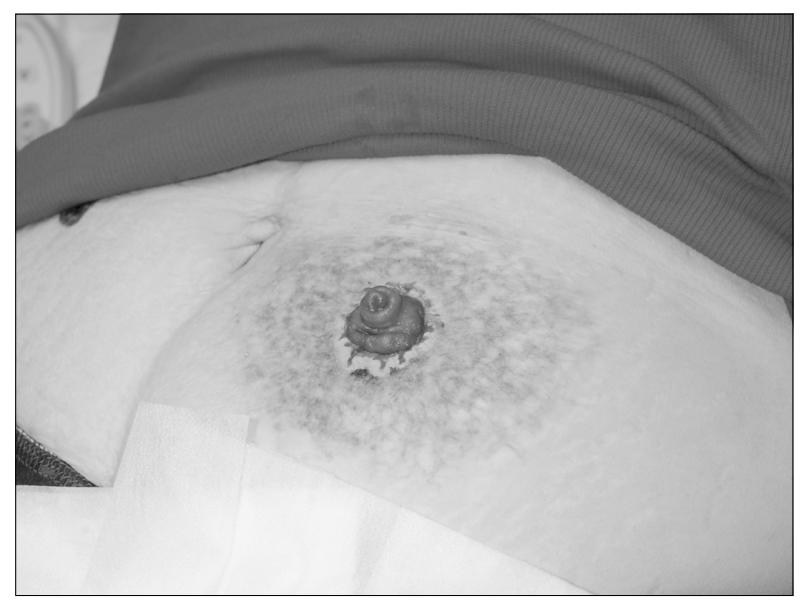

Fig. 1. Parastomal varices in patient 1. Abnormal radial vascular formation in surrounding skin with extensive circumferential purplish discolouration (raspberry appearance) and ulceration at the mucocutaneous junction of the ileostomy. he was diagnosed with primary sclerosing cholangitis (PSC) leading to development of liver cirrhosis, portal hypertension with associated multiple thromboses in the mesenteric and hepatic veins. The cirrhotic liver showed an irregular and nodular pattern on the CT scan (Fig. 2). He denied any history of excessive alcohol consumption. His chronic liver disease was classified as Child-Pugh class B.

He developed purplish discolouration and abnormal vascular formation around the ileostomy associated with frequent bleeding from the stoma. The initial suspicion was that of parastomal pyoderma gangrenosum; however, the biopsies did not support this diagnosis. The histology of the biopsies from this area showed non-specific inflammatory cell infiltrates.

Over the following two years he had multiple hospital admissions due to significant, acute parastomal bleeding which was sometimes associated with collapse. The bleeding was fresh blood with clots requiring transfusion of multiple units of blood. The persistent bleeding points were noted to be from small defects at the skin edge of the stoma. The diagnosis of bleeding parastomal varices was made and confirmed by the presence of prominent abnormal parastomal vasculature on CT scans (Fig. 3). Suturing of bleeding vessels at the identified mucocutaneous junction defect did not stop the bleeding. He was managed with terlipressin when particularly severe episodes of bleeding occurred.

This patient has since undergone multivisceral transplantation of the stomach, duodenum, small intestine, colon,

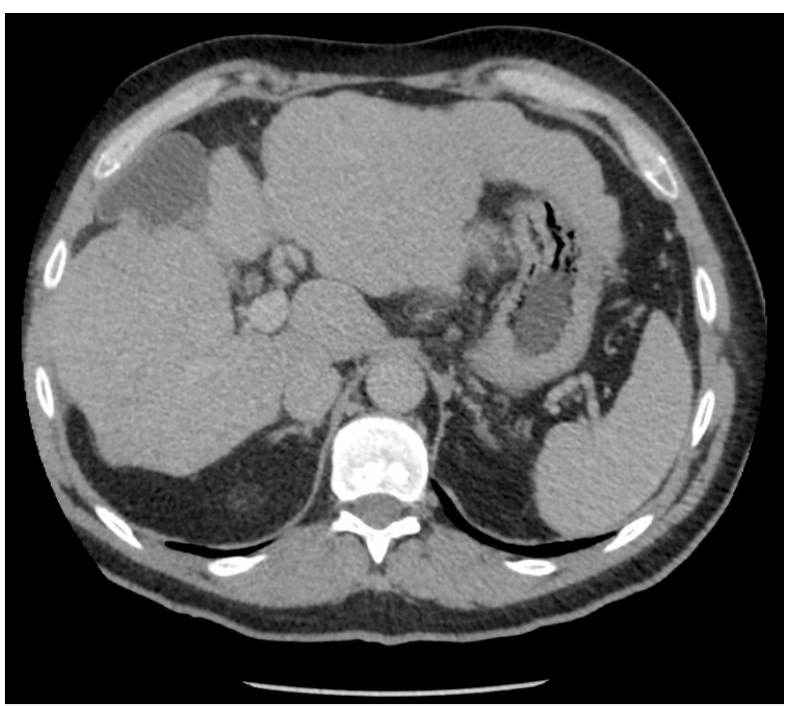

Fig. 2. CT scan showing a cirrhotic liver due to primary sclerosing cholangitis in patient 2. Irregular and nodular cirrhotic liver with portal hypertension. 


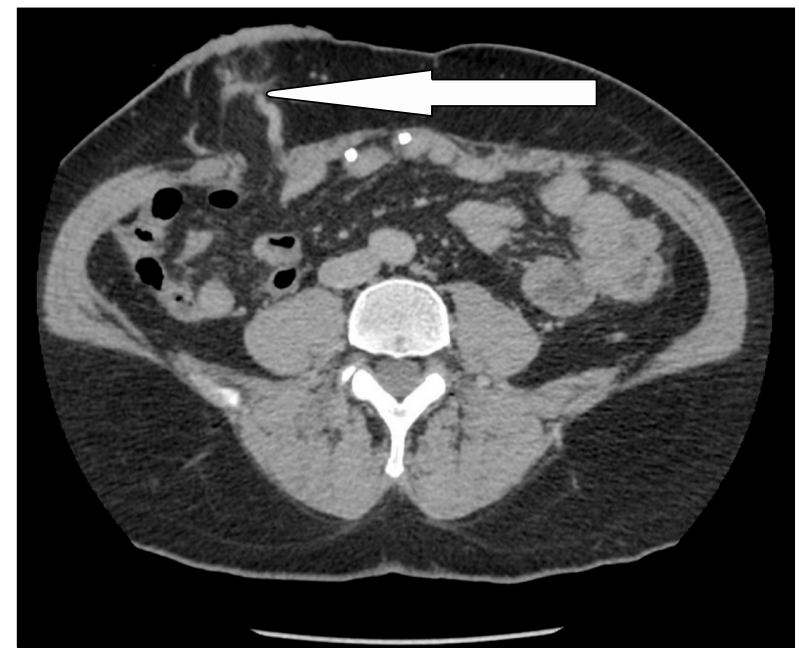

Fig. 3. CT scan showing parastomal variceal vessels in patient 2 . Presence of prominent and tortuous abnormal parastomal vasculature due to parastomal varices (collateral vessels) indicated with an arrow.

liver, and pancreas. He also underwent a splenectomy and an end colostomy formation.

\section{Case 3}

An 80-year-old man who has had excess alcohol consumption for over 30 years was diagnosed with alcoholic liver cirrhosis and portal hypertension in May 2009. He underwent an anterior resection and an end left iliac fossa colostomy in 2005 with preservation of his anal canal for a mid-rectal cancer.

Over the last 18 months, he presented on multiple occasions with purplish colouration around his end colostomy with recurrent bleeding from ulcerations from the edge of the stoma. The bleeding was severe on a few occasions requiring admission to hospital and blood transfusion.

On clinical examination, purplish circumferential colouration (raspberry appearance) was observed around the left iliac fossa stoma with three areas of ulcerations at the mucocutaneous junction with bleeding (Fig. 4). The diagnosis of parastomal varices was considered. His previous CT scan of the abdomen had confirmed irregular and nodular liver cirrhosis, oesophageal varices, and ascites. He was classified as Child-Pugh grade $B$.

He was advised to stop drinking alcohol and received various local treatments for the recurrent peristomal bleeding, including silver nitrate stick treatment and diathermy cauterisation, with no great success. He has had a trial with propranolol tablets with reduction in the frequency and severity of bleeding from peristomal varices.

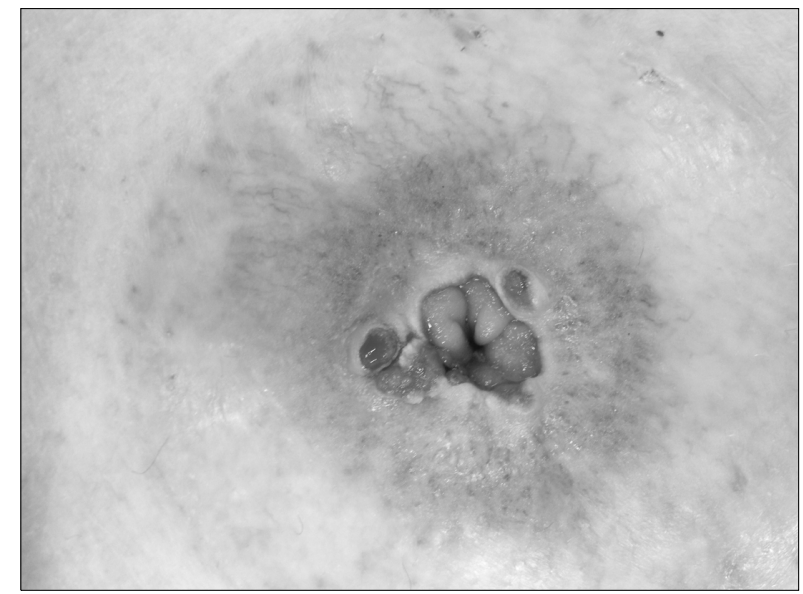

Fig. 4. Parastomal varices in patient 3. Purplish circumferential colouration (raspberry appearance) around the left iliac fossa stoma with three areas of ulcerations at the mucocutaneous junction and bleeding point.

\section{DISCUSSION}

Ectopic parastomal variceal formation is a rare condition in patients with chronic liver disease. Acute parastomal variceal bleeding can be life-threatening, challenging, and its diagnosis and management may be difficult. It was first documented in 1968 by Resnick et al. ${ }^{6}$ The functional anatomy of portosystemic shunting between the parietal surfaces of the abdominal viscera and the posterior abdominal wall has previously been well demonstrated. ${ }^{7}$ It has been postulated that peristomal varices, an example of ectopic portosystemic shunts may develop within the intra-abdominal adhesions of the abdominal wall around the site of the enterocutaneous stoma due to direct contact of the mesenteric (portocaval) circulation with the systemic circulation of the posterior abdominal wall. ${ }^{8}$

Parastomal variceal bleeding, like any other varices, is thought to be the result of portal hypertension resulting from chronic liver disease of various aetiologies. Liver cirrhosis is the most common cause of portal hypertension leading to development of parastomal varices, followed by PSC. ${ }^{9}$

Wiesner et al. ${ }^{10}$ reported the risk factors for development of peristomal varices, including oesophageal varices, advanced histological stage of PSC at liver biopsy, splenomegaly, hepatomegaly, increased serum bilirubin, decreased serum albumin, and decreased platelet count. The differential and pathologically high pressure between the portal vein and inferior vena cava results in formation of portosystemic collateral vessels that bypass part of the portal circu- 
lation to the systemic circulation.

Bleeding is the most common presentation of varices and portal hypertension and occurs mainly from the oesophagogastric varices. Acute recurrent bleeding from the mucocutaneous junction of the stoma may be severe and life threatening, as demonstrated by our three patients. Diagnosis of parastomal bleeding and its aetiology can be very challenging. The approach to diagnosis should be systematic through a comprehensive history looking for the risk factors of chronic liver disease. ${ }^{10}$ A careful physical examination for stigmata of chronic liver disease is essential to arriving at the diagnosis. The uncovered stomal site should be examined and the findings well documented in the clinical note. Diagnosis of the underlying chronic liver disease and features of portal hypertension can be achieved using an ultrasound scan, CT, and/or MRI scans. Doppler ultrasound scan and CT/MRI angiography are useful in identification of parastomal variceal bleeding., 2,40-12

Treatment of parastomal variceal bleeding is controversial and challenging too. Various treatment options have been reported in the literature $\mathrm{e}^{2,3,5,8-14}$ including local wound care and pressure dressings, suture ligation, and cautery with sclerosants as the first-line therapy. Sclerosing agents such as oily phenol, polidocanol, and cyanoacrylate are injected into the troublesome bleeding veins promoting vascular and perivascular inflammation and thrombosis of the vessels. Use of oral propranolol, a beta-blocker titrated against resting pulse has been widely recommended. ${ }^{9-11,13,14}$ Use of beta-blocker therapy has been well demonstrated to decrease the risk of first bleed in patients with oesophageal varices and mortality in patients with recurrent gastro-oesophageal variceal bleeding. ${ }^{9,14}$ Other commonly used pharmacotherapy includes vasoconstrictor such as terlipressin.

The effects of local and pharmacotherapy are usually short lived and patients will often continue to present with recurrent parastomal variceal bleeding. Closure of the stoma is ideal, where possible, or the stoma may be re-sited, but patients may soon develop similar pathology at the new stomal site. Surgical interventions such as shunting operation (transjugular intrahepatic portosystemic shunt) and liver transplantation are other available treatment options which may provide more definitive long term results but are associated with significant mortality and morbidity. 4,5,8-11,13,14

Parastomal variceal bleeding secondary to portal hypertension is rare but potentially life-threatening. The three pa- tients described here illustrate the diagnostic and management challenges of this entity. Primary prevention of parastomal variceal bleeding requires avoidance of enterocutaneous stomas in patients with portal hypertension if possible. Construction of an ileoanal pouch may enable avoidance of stomal formation, the attendant variceal shunting and bleeding in patients with PSC after total proctocolectomy for ulcerative colitis. Clinicians should maintain a high index of suspicion in patients with underlying chronic liver disease who present with recurrent parastomal bleeding. Immediate management must be aimed at controlling active bleeding and subsequently to lowering of portal venous pressures.

\section{REFERENCES}

1. Krige JE, Beckingham IJ. ABC of diseases of liver, pancreas, and biliary system. Portal hypertension-1: varices. BMJ 2001;322: 348-351.

2. Helmy A, Al Kahtani K, Al Fadda M. Updates in the pathogenesis, diagnosis and management of ectopic varices. Hepatol Int 2008;2:322-334.

3. Conte JV, Arcomano TA, Naficy MA, Holt RW. Treatment of bleeding stomal varices. Report of a case and review of the literature. Dis Colon Rectum 1990;33:308-314.

4. Norton ID, Andrews JC, Kamath PS. Management of ectopic varices. Hepatology 1998;28:1154-1158.

5. Farquharson AL, Bannister JJ, Yates SP. Peristomal varices--life threatening or luminal? Ann R Coll Surg Engl 2006;88:W6-W8.

6. Resnick RH, Ishihara A, Chalmers TC, Schimmel EM. A controlled trial of colon bypass in chronic hepatic encephalopathy. Gastroenterology 1968;54:1057-1069.

7. Edwards EA. Functional anatomy of the porta-systemic communications. AMA Arch Intern Med 1951:88:137-154.

8. Lebrec D, Benhamou JP. Ectopic varices in portal hypertension. Clin Gastroenterol 1985;14:105-121.

9. Pennick MO, Artioukh DY. Management of parastomal varices: who re-bleeds and who does not? A systematic review of the literature. Tech Coloproctol 2013;17:163-170.

10. Wiesner RH, LaRusso NF, Dozois RR, Beaver SJ. Peristomal varices after proctocolectomy in patients with primary sclerosing cholangitis. Gastroenterology 1986;90:316-322.

11. Spier BJ, Fayyad AA, Lucey MR, et al. Bleeding stomal varices: case series and systematic review of the literature. Clin Gastroenterol Hepatol 2008;6:346-352.

12. Choi JW, Lee CH, Kim KA, Park CM, Kim JY. Ectopic varices in coIonic stoma: MDCT findings. Korean J Radiol 2006;7:297-299.

13. Kabeer MA, Jackson L, Widdison AL, Maskell G, Mathew J. Stomal varices: a rare cause of stomal hemorrhage. A report of three cases. Ostomy Wound Manage 2007;53:20-22, 24, 26 passim.

14. Wilbur K, Sidhu K. Beta blocker prophylaxis for patients with variceal hemorrhage. J Clin Gastroenterol 2005;39:435-440. 